CASE REPORT

\title{
Regression of idiopathic thrombocytopenic purpura after endoscopic mucosal resection of gastric mucosa associated lymphoid tissue lymphoma
}

\author{
M Noda, N Mori, K Nomura, K Kojima, S Mitsufuji, I Yamane, S Misawa, T Okanove
}

Gut 2004;53:1698-1700. doi: 10.1136/gut.2003.033555

Recent reports have suggested an association between Helicobacter pylori infection and both gastric mucosa associated lymphoid tissue (MALT) lymphoma and thrombocytopenic purpura. Although treatments eradicating $\mathrm{H}$ pylori lead to regression of these diseases in some cases, the exact mechanisms are still controversial. This case report describes a patient with thrombocytopenic purpura accompanied by an early stage gastric MALT lymphoma. Endoscopic mucosal resection of the lesion in this patient led to dramatic regression of thrombocytopenic purpura, and $t(11 ; 18)(q 21 ; q 21)$, which means resistance more likely to $\mathrm{H}$ pylori eradication therapy, was confirmed by fluorescence in situ hybridisation. There is no evidence of recurrence and his platelet count is within normal limits after 24 months of follow up. This is the first case report describing regression of thrombocytopenic purpura after mucosal resection of a gastric MALT lymphoma. We suggest that while some cases of thrombocytopenic purpura may be induced by $\mathrm{H}$ pylori, others may be due to an autoreactive antibody produced by MALT lymphoma B cells.

M alignant lymphomas or mucosa associated lymphoid tissue (MALT) lymphomas are occasionally accompanied by thrombocytopenic purpura, suggesting an immunological origin for thrombocytopenic purpura. Treatments eradicating Helicobacter pylori lead to regression of these diseases in some cases, and evidence points to an important role for $H$ pylori infection in the development of idiopathic thrombocytopenic purpura (ITP). ${ }^{23}$ We present here a very rare but intriguing case of gastric MALT lymphoma that showed regression of thrombocytopenic purpura after endoscopic mucosal resection (EMR), and in which $\mathrm{t}(11 ; 18)(\mathrm{q} 21 ; \mathrm{q} 21)$ was confirmed by fluorescence in situ hybridisation.

\section{CASE REPORT}

A 65 year old man was referred to our department in August 2001 with a one month history of decreased appetite and epigastric pain. He had a past history of non-insulin dependent diabetes mellitus, hyperlipidaemia, and an old myocardial infarction. Upper gastrointestinal endoscopy showed an $8 \mathrm{~mm}$ irregular erosion on the greater curvature of the fornix. Differential diagnosis of early gastric cancer IIc (superficial depressed) type was difficult but the histological diagnosis was gastritis with erosive change. The patient was admitted to our department for further examination and therapy because an endoscopic observation after two months demonstrated a $12 \mathrm{~mm}$ submucosal tumour-like lesion with a shallow ulcer on the surface (fig 1).
On admission, physical examination was unremarkable except for mild epigastric tenderness; the liver, spleen, and superficial lymph nodes were not palpable. His blood cell count however showed a platelet level of $27000 / \mathrm{mm}^{3}$. Other routine blood chemistry, liver function tests, chest radiography, and urinalysis were normal. Although we stopped administering diltiazem, famotidine, and atrovastatin to eliminate drug induced thrombocytopenia, his platelet level did not recover. A bone marrow smear showed normal cellularity and an increased number of megakaryocytes. Antiplatelet antibody titres (IgG), as tested by indirect immunofluorescence, were increased. No abnormalities in blood coagulation or in levels of autoimmune antibodies were observed.

When his platelet count was $40000-50000 / \mathrm{mm}^{3}$, we obtained informed consent and performed EMR using a two channel scope (GIF-2T200; Olympus, Tokyo, Japan) to make an accurate histological diagnosis. ${ }^{45}$ Five days after EMR, his platelet count increased dramatically to $173000 / \mathrm{mm}^{3}$ (fig 2). Specimens were fixed in $10 \%$ formalin, embedded in paraffin, and routinely stained with haematoxylin and eosin (H\&E). They were also sectioned for immunohistochemical study and for tissue fluorescence in situ hybridisation (T-FISH). ${ }^{6}$ The lesion was composed of diffuse small atypical lymphoid cells with centrocyte-like appearance (cleaved nucleus and relatively wide clear cytoplasm) that had invaded the lamina propria. The atypical lymphoid cells produced characteristic lymphoepithelial lesions, and immunohistochemically they were diffusely positive for CD20 but negative for CD5 and CD10. By T-FISH analysis it was positive for $\mathrm{t}(11 ; 18)(\mathrm{q} 21 ; \mathrm{q} 21)$ but negative for trisomy 3,7 , 12 , and 18 (fig 3).

Ultrasonography and computed tomography showed no hepatosplenomegaly or swelling of the para-aortic lymph nodes. The tumour was diagnosed as a stage 1 extranodal marginal zone MALT B cell lymphoma. Because the rapid urease test and biopsy specimens from the gastric body and antrum were positive for $H$ pylori, the patient was treated with a seven day course of lansoprazol $60 \mathrm{mg}$ twice a day, amoxicillin $1500 \mathrm{mg}$ twice a day, and clarithromycin $400 \mathrm{mg}$ twice a day, one month after EMR. He did not receive antibiotics at any time except for this period. Three months after treatment, gastric biopsy specimens were negative for lymphoma infiltration and $H$ pylori, by histology and the rapid urease test, and the patient tested negative after a urea breath test. No additional therapy, such as chemotherapy or radiation, was performed. There is no evidence of recurrence

Abbreviations: MALT, mucosa associated lymphoid tissue; ITP, idiopathic thrombocytopenic purpura; $E M R$, endoscopic mucosal resection; H\&E, haematoxylin and eosin; T-FISH, tissue fluorescence in situ hybridisation 

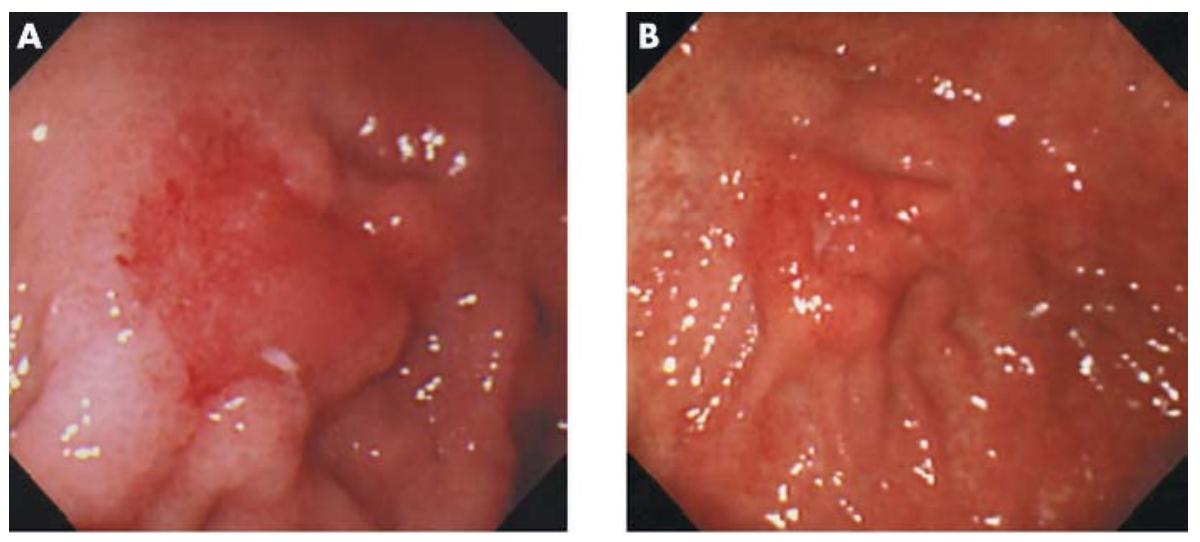

Figure 1 (A) Endoscopic observation of the stomach showed a slightly elevated irregular erosion at the greater curvature of the fornix. (B) Endoscopic observation after two months revealed a $12 \mathrm{~mm}$ submucosal tumour-like lesion with a shallow ulcer on the surface.

and his platelet count is within normal limits after 24 months of follow up after endoscopic removal.

\section{MATERIALS AND METHODS}

Lymphoma specimens were fixed in $10 \%$ formalin, embedded in paraffin, and stored at room temperature. Sections $(4 \mu \mathrm{m}$ thick) were cut from paraffin embedded tissue and used for histopathological observation and T-FISH. The former was performed according to routine procedures for $\mathrm{H} \& \mathrm{E}$ and Giemsa stainings and the latter was performed as described previously. ${ }^{6}$ For detection of $\mathrm{t}(11 ; 18)(\mathrm{q} 21 ; \mathrm{q} 21)$, we used yeast artificial chromosome clones y943b8 (specific for 18q21) and y966e4 (specific for 1lq21) (obtained from MIT Center for Genome Research, Cambridge, Massachusetts, USA). Polymerase chain reaction products were labelled with either SpectrumGreen (Vysis Inc., Illinois, USA) or SpectrumOrange (Vysis Inc.) with a nick translation kit (Vysis Inc.). For detection of trisomy 3, 7, 12, and 18, we used CEP Probes CEP3 (D3Z1), CEP7 (D7Z1), CEP12 (D12Z3), and CEP18 (D18Z1) SpectrumOrange (Vysis Inc.), respectively.

Each nucleus was identified on the basis of 6-diaminido-2phenylindole dihydrochloride staining. Images were captured with a CCD camera. Signals from at least 200 non-overlapping nuclei were evaluated per slide. For T-FISH analysis, we assessed only colocalised signals. To evaluate colocalised signals, cut off values were calculated from the mean $(+2$ $\mathrm{SD})$, which were based on evaluation of 100 nuclei in each specimen from three patients with chronic gastritis.

\section{DISCUSSION}

MALT lymphomas are considerably mature B cell lymphomas of low malignancy that were first described by Isaacson and Wright in 1983. They were classified as marginal zone peripheral B cell lymphomas deriving from marginal zone

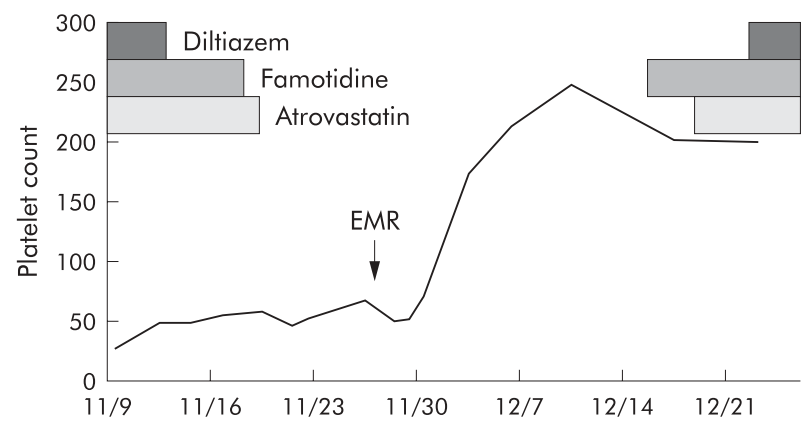

Figure 2 Dramatic increase in platelet count after endoscopic mucosal resection (EMR). Suspension or re-administration of diltiazem, famotidine, and atrovastatin did not affect the platelet level. cells at extranodal-extrasplenic sites in the World Health Organization classification. They are composed of B cell clones expanded by activation of antigen sensitive T cells, and in the case of gastric MALT lymphomas, the major antigen results from $H$ pylori infection. Most MALT lymphomas induced by $H$ pylori infection can be treated by eradication therapy $^{7}$ but there are some that are resistant. These MALT lymphomas resistant to eradication therapy are associated with the presence of API2/MALT1 chimeric gene by $t(11 ; 18)$ (q21; 21 ) translocation. ${ }^{8-10}$ This translocation has been found in approximately $50 \%$ of MALT lymphomas and in $10-20 \%$ of gastric MALT lymphomas. ${ }^{11}$

ITP is considered to be autoimmune thrombocytopenia associated with production of antiplatelet antibodies, the underlying diseases or causes of which are not known. It is also defined as acquired thrombocytopenia that causes haemorrhagic tendency in the skin or submucosa despite normal or overactive platelet production. As standard therapy, use of corticosteroids as immune suppressive therapy is recommended in the first instance, and splenectomy has been recommended for ineffective cases. Recently, however, some improvement in ITP by $H$ pylori eradication therapy has been observed subsequent to the initial report by Gasbarrini et al in $1998 .{ }^{2}$ In these reports, $58 \%$ of ITP patients were found to be positive for $H$ pylori infection, and $21-24 \%$ of ITP patients showed a consistent and lasting increase in platelet counts following $H$ pylori eradication therapy. ${ }^{3}$

$H$ pylori infection is thought to induce an immunological response resulting in production of antiplatelet antibodies but the exact mechanism of the $H$ pylori action is unclear. Interestingly, it has been determined that the antibody produced by MALT lymphoma B cells recognises a variety of autoantigens without cross reactivity to $H$ pylori. ${ }^{12}$ In an analysis of the CDR3 region that codes for the antigen specificity of an antibody, it was revealed that the antibodies produced by MALT B cell lymphoma had exhibited similarities to autoimmune antibodies such as antinuclear antibodies. ${ }^{13}$ Furthermore, Hussell et al reported that proliferation of low grade B cell MALT lymphomas of the stomach depends on the presence of tissue infiltrating T cells. ${ }^{14}$ Because ITP showed regression after EMR of MALT lymphoma without $H$ pylori eradication in the present case, ITP could be induced by an antibody produced by MALT lymphoma B cells. Our patient received anti- $H$ pylori therapy one month after EMR because antibiotics are accepted as firstline therapy for gastric MALT lymphoma. ${ }^{15}$ However, an increased platelet count was observed five days after EMR, indicating that regression of thrombocytopenia was independent of $H$ pylori eradication therapy, and was instead the result of the EMR itself.

Generally, most gastric MALT lymphomas are closely linked to chronic $H$ pylori infection, and $60-80 \%$ of low grade 

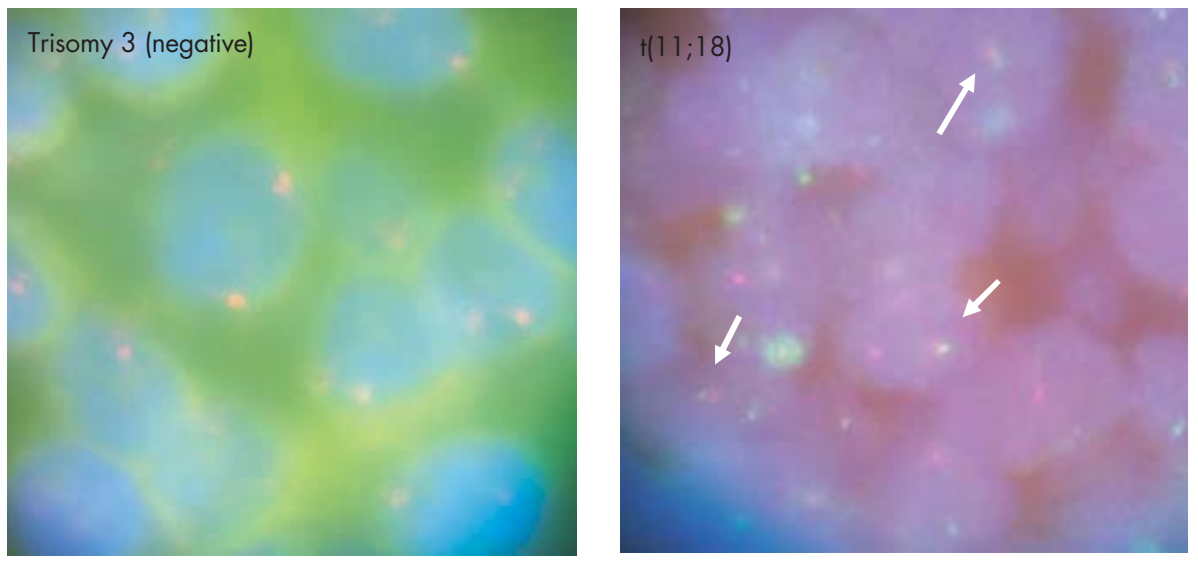

Figure 3 Tissue fluorescence in situ hybridisation findings on paraffin embedded tissue section. Red signals are representative of $18 q 21$ whereas green ones are of $11 \mathrm{q} 21$. Arrows indicate colocalised signal $(\times 1000)$.

MALT lymphomas regress in response to $H$ pylori eradication therapy. In this case, however, molecular biology confirmed $\mathrm{t}(11 ; 18)(\mathrm{q} 21 ; \mathrm{q} 21)$ of the lesion, as detected by the T-FISH method, indicating a more likely resistance to $H$ pylori eradication therapy. To date, radiation therapy, surgical resection, or chemotherapy have often been recommended for such cases but we were able to treat the MALT lymphoma with EMR and there was no evidence of recurrence after 24 months of follow up. Furthermore, ITP also regressed after endoscopic removal of the MALT lymphoma.

In conclusion, this is the first case report on regression of thrombocytopenic purpura after mucosal resection of a gastric MALT lymphoma. The mechanisms of regression of ITP remain to be clarified but it appears that ITP may not be directly induced by $H$ pylori infection in some cases but by an antibody produced by MALT lymphoma B cells or proliferated $\mathrm{B}$ cells in response to $H$ pylori infection via $\mathrm{T}$ cell help.

\section{ACKNOWLEDGEMENTS}

This work was supported in part by: (1) Grant-in-Aid for Young Scientists (B) from the Ministry of Education, Culture, Sports, Science and Technology of Japan (13770268) and (2) supporting research funds from the Japanese Foundation for Research and Promotion of Endoscopy (JFE-2002).

\footnotetext{
Authors' affiliations

M Noda, K Kojima, I Yamane, Department of Gastroenterology, Kyoto

Prefectural Yosanoumi Hospital, Kyoto, Japan, and Molecular Gastroenterology and Hepatology, Kyoto Prefectural University of Medicine Graduate School of Medical Science, Kyoto, Japan

N Mori, Department of Gastroenterology, Kyoto Prefectural Yosanoumi Hospital, Kyoto, Japan

K Nomura, Molecular Haematology and Oncology, Kyoto Prefectural University of Medicine Graduate School of Medical Science, Kyoto, Japan

S Mitsufuii, Molecular Gastroenterology and Hepatology, Kyoto

Prefectural University of Medicine Graduate School of Medical Science, Kyoto, Japan

S Misawa, Department of Gastroenterology, Kyoto Prefectural Yosanoumi Hospital, Kyoto, Japan, and Molecular Haematology and Oncology, Kyoto Prefectural University of Medicine Graduate School of Medical Science, Kyoto, Japan

T Okanove, Molecular Gastroenterology and Hepatology, and Molecular Haematology and Oncology, Kyoto Prefectural University of Medicine Graduate School of Medical Science, Kyoto, Japan
}

Correspondence to: Dr M Noda, Department of Gastroenterology, Kyoto Prefectural Yosanoumi Hospital, Otokoyama 481, Iwataki-cho, Yosa-gun, Kyoto 629-2261, Japan; mnoda@mbox.kyoto-inet.or.jp

Revised version received 2 April 2004

Accepted for publication 13 April 2004

\section{REFERENCES}

1 Bachmeyer C, Audouin J, Bouillot JL, et al. Immune thrombocytopenic purpura as the presenting feature of gastric MALT lymphoma. Am J Gastroenterol 2000;95:1599-600

2 Gasbarrini A, Franceschi F, Tartaglione R, et al. Regression of autoimmune thrombocytopenia after eradication of Helicobacter pylori. Lancet 1998;352:878.

3 Emilia G, Luppi M, Morselli M, et al. Helicobacter pylori infection and idiopathic thrombocytepenic purpura. Br J Haematol 2002;1 18:1198-9.

4 Noda M, Kodama T, Atsumi M, et al. Possibilities and limitations of endoscopic resection for early gastric cancer. Endoscopy 1997;29:361-5.

5 Noda M, Kobayashi N, Kanemasa H, et al. Endoscopic mucosal resection using a partial transparent hood for lesions located tangentially to the endoscope. Gastrointest Endos 2000;51:338-43.

6 Nomura K, Yoshino T, Nakamura S, et al. Detection of $t(11 ; 18)(q 21 ; q 21)$ in marginal zone lymphoma of mucosa-associated lymphocystic tissue type on paraffin-embedded tissue sections by using fluorescence in situ hybridization. Can Genet Cytogenet 2003; 140:49-54.

7 Wotherspoon AC, Doglioni C, Diss TC, et al. Regression of primary low-grade B-cell gastric lymphoma of mucosa-associated lymphoid tissue type after eradication of Helicobacter pylori. Lancet 1993;342:575-7.

8 Akagi T, Motegi $M$, Tamura A, et al. A novel gene, MALT1 at 18q21, is involved in $\uparrow(11 ; 18)(q 21 ; q 21)$ found in low-grade B-cell lymphoma of mucosa-associated lymphoid tissue. Oncogene 1999;18:5785-94.

9 Alpen B, Neubauer A, Dierlamm J, et al. Translocation $t(11 ; 18)$ absent in early gastric marginal zone B-cell lymphomas of MALT type responding to eradication of Helicobacter pylori infection. Blood 2000;95:4014-15.

10 Liu H, Ruskon-Fourmestraux A, Lavergne-Slove A, et al. Resistance of $t(11 ; 18)$ positive mucosa-associated lymphoid tissue lymphoma to Helicobacter pylori eradication therapy. Lancet $2001 ; 357: 39-40$.

11 Nakamura T, Nakamura S, Yokoi T, et al. Clinico-pathological comparison between the API2-MALT1 chimeric transcript-positive and -negative gastric low-grade B-cell lymphoma of mucosa-associated lymphoid tissue type. Jpn J Cancer Res 2002;93:677-84.

12 Hussell T, Isaacson PG, Crabtree JE, et al. Immunoglobulin specificity of low grade $B$ cell gastrointestinal lymphoma of mucosa-associated lymphoid tissue (MALT) type. Am J Pathol 1993;142:285-92.

13 Yumoto N, Kurosu K, Furukawa M, et al. CDR3 sequences of MALT lymphoma show homology with those of autoreactive B-cell lines. Jpn J Cancer Res 1999;90:849-57.

14 Hussell T, Isaacson PG, Crabtree JE, et al. The response of cells from lowgrade B-cell gastric lymphomas of mucosa-associated lymphoid tissue to Helicobacter pylori. Lancet 1993;342:571-4.

15 Caletti G, Togliani T, Fusaroli $P$, et al. Consecutive regression of concurrent laryngeal and gastric MALT lymphoma after anti-Helicobacter pylori therapy. Gastroenterology 2003;124:537-43. 